\title{
Prognostic Implications of Progressive Cardiac Conduction Disease
}

\author{
Tamiro Kawaguchi, MD; Hideki Hayashi, MD, PhD; Akashi Miyamoto, MD, PhD; \\ Tomohide Yoshino, MD; Atsushi Taniguchi, MD; Nobu Naiki, MD; \\ Yoshihisa Sugimoto, MD, PhD; Makoto Ito, MD, PhD; Joel Q. Xue, PhD; \\ Yoshitaka Murakami, PhD; Minoru Horie, MD, PhD
}

\begin{abstract}
Background: Progressive cardiac conduction disease (PCCD), characterized by temporal increase in PR interval and QRS duration, may be attributed to diverse pathophysiological mechanisms. This study aimed to investigate whether PCCD is associated with increased risk of cardiovascular morbidity and mortality.
\end{abstract}

\begin{abstract}
Methods and Results: Digital analysis of 12-lead ECG was performed to select patients with PCCD from among a database containing 359,737 ECGs. Long-term prognosis of PCCD was assessed in a large hospital-based population: 458 patients (341 males; mean age, 57.9 \pm 14.7 years) with PCCD were enrolled. During a mean followup of $13.3 \pm 6.4$ years, 109 patients were hospitalized for heart failure (HF), and there were 16 and 59 deaths from cardiovascular diseases and all causes, respectively. Multivariate Cox proportional hazards analysis confirmed (1) a significant association of temporal incremental rate of PR interval ( $\geq 2 \mathrm{~ms} / \mathrm{year}$ ) and QRS duration ( $\geq 3 \mathrm{~ms} / \mathrm{year}$ ) with $\mathrm{HF}$ hospitalization (hazard ratio [HR], 2.34; 95\% confidence interval [Cl], 1.36-4.05; $\mathrm{P}=0.002$ and $\mathrm{HR}, 2.08 ; 95 \% \mathrm{Cl}$, 1.25-3.53; $P=0.01$, respectively) and (2) a significant association of temporal incremental rate of $P R$ interval ( $\geq 4 \mathrm{~ms} /$ year) and QRS duration ( $\geq 5 \mathrm{~ms} /$ year) with cardiovascular mortality $(\mathrm{HR}, 6.9 ; 95 \% \mathrm{Cl}, 1.47-36.96 ; \mathrm{P}=0.02$ and $\mathrm{HR}, 4.31 ; 95 \% \mathrm{Cl}, 1.19-16.5 ; \mathrm{P}=0.03$, respectively).
\end{abstract}

Conclusions: The severity of PCCD was independently and significantly associated with HF hospitalization and cardiovascular mortality. (Circ J 2013; 77: 60-67)

Key Words: Conduction; Electrocardiography; Heart failure; Prognosis; Ventricles

$\mathbf{P}$ rogressive cardiac conduction disease (PCCD) is characterized by electrical deterioration of the conduction system in the atrium and ventricle, thus presenting as temporal prolongation of the PR interval and QRS duration. Lev and Unger ${ }^{1}$ and Lenègre ${ }^{2}$ reported pathological abnormalities in the conduction system with deposition of fibrous tissue in patients who developed ventricular conduction disturbance. On the other hand, inherited arrhythmias, including sick sinus syndrome, atrioventricular block and bundle branch block, have been reported. Several genetic abnormalities have been found in patients with inherited cardiac conduction abnormalities, ${ }^{3}$ but although these disorders exhibit remarkable clinical characteristics such as syncope, sudden death and pacemaker implantation requirement, the prevalence is rare.

\section{Editorial p 41}

In contrast, the relation of QRS duration with an increased rate of mortality 4,5 and reduced left ventricular ejection function $^{6}$ has been studied in numerous patients with and without ischemic heart diseases. In patients with myocardial infarction and reduced cardiac function, the benefit from implantablecardioverter defibrillator therapy is higher when the QRS duration is longer. ${ }^{7}$ These findings suggest that PCCD plays an important role in determining the prognosis of various cardiac disorders, including ischemic and nonischemic heart diseases.

However, little is known about the effect of temporal deterioration of supra- and intraventricular conduction on prognosis in a large hospital-based population. In Shiga University of Medical Science's hospital, more than 350,000 ECGs obtained from more than 100,000 patients are available for digital analysis. The long-term outcomes are precisely identified by the medical records. Using this large database, we systematically examined the association of PCCD with cardiovascular morbidity and mortality. This study also benefited from reproducible ECG measures based on computer-processed analyses.

Received July 2, 2012; revised manuscript received August 3, 2012; accepted August 23, 2012; released online September 26, 2012 Time for primary review: 8 days

Department of Cardiovascular and Respiratory Medicine (T.K., H.H., A.M., T.Y., A.T., N.N., Y.S., M.I., M.H.), Department of Medical Statistics (Y.M.), Shiga University of Medical Science, Otsu, Japan; and General Electric Healthcare, Milwaukee, WI (J.Q.X.), USA

Mailing address: Hideki Hayashi, MD, PhD, Department of Cardiovascular and Respiratory Medicine, Shiga University of Medical Science, Otsu 520-2192, Japan. E-mail: hayashih@ belle.shiga-med.ac.jp

ISSN-1346-9843 doi:10.1253/circj.CJ-12-0849

All rights are reserved to the Japanese Circulation Society. For permissions, please e-mail: cj@j-circ.or.jp 


\section{Methods}

The research protocol was approved by the Ethical Committee of Shiga University of Medical Science.

\section{Study Population}

In the University's hospital, digital recording of 12-lead ECGs started in January 1983. Until July 2010, a total of 114,334 consecutive patients $(55,091$ females and 59,243 males) underwent 12-lead ECG recordings in the supine position. The ECG database comprises a total of 359,737 ECGs collected during that period. The 12-lead ECG was recorded at rest for $10 \mathrm{~s}$ at a sweep speed of $25 \mathrm{~mm} / \mathrm{s}$, calibrated to $1 \mathrm{mV} / \mathrm{cm}$ in the standard leads. The data were digitally stored in a 12-bit server computer with a sampling interval of $2 \mathrm{~ms}$.

From the database, we chose patients who exhibited wide QRS duration $\geq 120$ ms between January 2000 and December 2003, enrolled in this study with the last follow-up on December 2010. To determine the temporal increase in QRS duration, the time interval of serial ECG recordings was at least $\geq 1$ year. ECGs exhibiting Wolff-Parkinson-White syndrome, ventricular pacing, junctional or idioventricular rhythm and ventricular tachyarrhythmias were excluded. Patients who were $<15$ years old were also excluded from the analysis.

\section{ECG Analysis}

The ECG analysis was performed using software (MUSE7.1, GE Marquette Medical Systems, Inc, Milwaukee, WI, USA). Standardized, computerized ECG criteria as described by a 12-lead ECG analysis program were used to diagnose abnormal intraventricular morphologies. ECG variables, including duration, interval, amplitude, and axis, were digitally measured. For the ECG measurement, a median complex was computed as follows: (1) all QRS complexes of the same morphology were aligned in time and (2) the algorithm generated a representative QRS complex from the median voltages that were found at each successive sample time. QRS duration was measured from the earliest detection of depolarization in any lead (QRS onset) to the latest detection of depolarization in any lead (QRS offset). PR interval was measured in a similar manner. Because all variables of the 12-lead ECG were digitally measured based on computer-processed analysis, neither intra- nor interobserver variability needed to be taken into account and all measures were reproducible.

\section{Follow-up}

The follow-up period of all patients started from the day of the first ECG recording. We explored the prognostic factors for the endpoints of this study: cardiovascular death, all-cause death, and heart failure (HF) hospitalization. The outcome was assessed by searching the medical records in the hospital database. The determination of HF hospitalization was based on review of the hospital records. HF hospitalization had to satisfy both of the following criteria: (1) admission to hospital for $\geq 24 \mathrm{~h}$ with a clinical history of worsening symptoms of $\mathrm{HF}$ as evidenced by clinical criteria, including increased New York Heart Association functional class, orthopnea, paroxysmal nocturnal dyspnea, edema, exertional dyspnea, or gastrointestinal symptoms attributable to $\mathrm{HF}$, and (2) one or more intensive treatments for HF within $24 \mathrm{~h}$ of admission, such as intravenous diuretics or inotropic agents. All ECGs taken during the follow-up were evaluated in patients enrolled in this study. The first ECG recording was assigned as the baseline ECG. The ECG recording in which the QRS duration was longest during follow-up was defined as the follow-up ECG.

\begin{tabular}{|lc|}
\hline \multicolumn{2}{|l|}{ Table 1. Baseline Demographics of the Patients } \\
Total patients, $\mathrm{n}$ & 458 \\
Age (years) & $57.9 \pm 14.7$ \\
Sex, male (\%) & $341(74.5)$ \\
Follow-up period of ECG (years) & $9.0 \pm 5.7$ \\
Survival period (years) & $13.3 \pm 6.4$ \\
HF, $\mathrm{n}(\%)$ & $42(9.2)$ \\
Ischemic heart disease, $\mathrm{n}(\%)$ & $55(12.0)$ \\
Cardiomyopathy, $\mathrm{n}(\%)$ & $14(3.1)$ \\
Hypertension, $\mathrm{n}(\%)$ & $160(34.9)$ \\
Diabetes mellitus, $\mathrm{n}(\%)$ & $96(21.0)$ \\
Valvular heart disease, $\mathrm{n}(\%)$ & $19(4.1)$ \\
AF, $\mathrm{n}(\%)$ & $29(6.3)$ \\
Arrhythmias*, $\mathrm{n}(\%)$ & $30(6.6)$ \\
Malignant disease, $\mathrm{n}(\%)$ & $32(7.0)$ \\
Cardiovascular surgery, $\mathrm{n}(\%)$ & $38(8.3)$ \\
Othert, $\mathrm{n}(\%)$ & $31(6.8)$ \\
\hline
\end{tabular}

Data are presented as mean \pm standard deviation and $n(\%)$. ${ }^{*}$ Arrhythmia involves patients with various types of rhythm disorder except for patients who exhibited AF when the ECG was recorded. tIncludes patients who suffered various internal diseases. $H F$, heart failure; $A F$, atrial fibrillation.

\section{Statistical Analysis}

We described continuous variables using mean and standard deviation, and categorical variables using number and percentage. Comparisons between groups were made by t-test for continuous variables and $\chi^{2}$ test for categorical variables. A receiver-operating characteristic curve was used to determine the cutoff point of prognostic factors that optimized the sensitivity and specificity of ECG variables for the endpoints. A Kaplan-Meier curve was created to describe the event-free survival rate and differences between groups were compared by log-rank test. Cox proportional hazards models were used to estimate hazard ratios of HF hospitalization, cardiovascular mortality and all-cause mortality adjusted by age, sex, and other confounding factors. Variables included in the Cox model were selected by a variable procedure with a criteria of $\mathrm{P}<0.1$ for exclusion. All tests were 2-tailed and the significance level was set at 0.05 . Because this study primarily evaluated cardiac conduction, we repeated the statistical analyses with patients without nodal-blocking agents such as $\beta$-blockers, VaughanWilliams classes I, III, and IV anti-arrhythmic drugs, digitalis, and $\mathrm{H} 2$ histamine-blocker.

\section{Results}

A total of 458 consecutive patients (341 men; mean age, $57.9 \pm 14.7$ years) were enrolled in this study. Among them, $109(23.8 \%)$ were hospitalized because of HF. In addition, 59 $(12.9 \%)$ patients died, of which cardiovascular death occurred in $16(3.5 \%)$ patients. The mean duration of follow-up was $13.3 \pm 6.4$ years.

\section{Clinical Characteristics of the Patients}

Table 1 shows the baseline clinical characteristics of the patients enrolled in this study. The study population consisted of patients with various cardiac diseases and other disorders including diabetes, malignancy, and surgery. The number of ECG recordings averaged $17.8 \pm 21$ (median: 11) per patient. Table 2 shows 12-lead ECG measures and morphological characteris- 
Table 2. Characteristics of 12-Lead ECGs

\section{First ECG Follow-up ECG Temporal change ${ }^{\star} \quad$ P value}

ECG measures

\begin{tabular}{lcccc} 
Heart rate (beats/min) & $68.6 \pm 14.1$ & $66.1 \pm 13.1$ & $-2.7 \pm 15.5$ & 0.01 \\
PR interval (ms) & $165.0 \pm 27.4$ & $178.5 \pm 34.0$ & $13.9 \pm 20.2$ & $<0.0001$ \\
QRS duration (ms) & $118.9 \pm 22.8$ & $142.9 \pm 15.4$ & $24.0 \pm 22.5$ & $<0.0001$ \\
QRS morphology & & & & \\
Normal (n, \%) & $143,31.2$ & 0,0 & -31.2 & $<0.0001$ \\
RBBB (n, \%) & $186,40.6$ & $297,64.8$ & 24.2 & $<0.0001$ \\
RBBB with LAH (n, \%) & $13,2.8$ & $37,8.1$ & 5.3 & 0.0004 \\
LBBB (n, \%) & $31,6.8$ & $47,10.3$ & 3.5 & 0.06 \\
NSIVCD (n, \%) & $85,18.6$ & $76,16.6$ & -2.0 & 0.43 \\
\hline
\end{tabular}

Data are presented as mean \pm standard deviation and $n(\%)$.

*Temporal change indicates the difference in ECG measures or the prevalence of QRS morphology between the baseline ECG and the follow-up ECG.

RBBB, right bundle branch block; LAH, left anterior hemiblock; LBBB, left bundle branch block; NSIVCD, nonspecific intraventricular conduction disturbance.
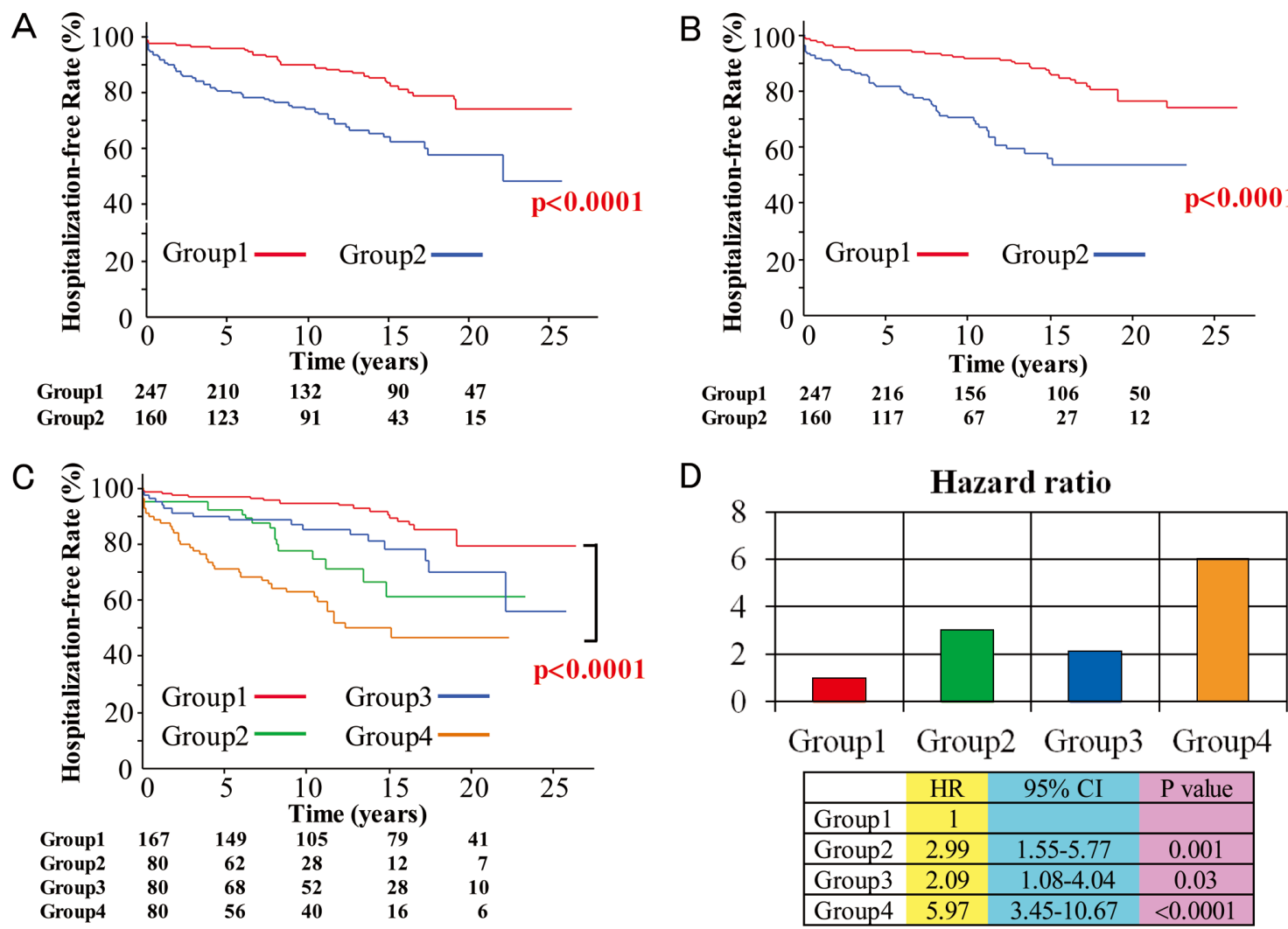

Figure 1. Kaplan-Meier survival analysis for a hospitalization for heart failure. (A) Temporal incremental rate of QRS duration $<3 \mathrm{~ms} /$ year (Group 1) vs. $\geq 3 \mathrm{~ms} /$ year (Group 2). (B) Temporal incremental rate of PR interval <2ms/year (Group 1) vs. $\geq 2 \mathrm{~ms} / \mathrm{year}$ (Group 2). (C) Classification into 4 groups based on temporal incremental rate of QRS duration and PR interval: Group 1 consists of patients with QRS-duration incremental rate $<3 \mathrm{~ms} /$ year and PR-interval incremental rate of $<2$ ms/year; Group 2, patients with QRS-duration incremental rate $<3 \mathrm{~ms} /$ year and PR-interval incremental rate $\geq 2 \mathrm{~ms} / \mathrm{year}$; Group 3, QRS-duration incremental rate $\geq 3 \mathrm{~ms} /$ year and PR-interval incremental rate $<2 \mathrm{~ms} / \mathrm{year}$; and Group 4, QRS-duration incremental rate $\geq 3 \mathrm{~ms} /$ year and PR-interval incremental rate $\geq 2 \mathrm{~ms} /$ year. (D) Interaction of hazard ratio of groups. Grouping is as in Panel C. Numbers of subjects at risk are shown under each graph. 
Table 3. Univariate and Multivariate Survival Analyses of HF Hospitalization

\begin{tabular}{|c|c|c|c|c|c|c|}
\hline & \multicolumn{2}{|c|}{$\begin{array}{l}\text { Univariate analysis } \\
\qquad(n=407)\end{array}$} & \multicolumn{2}{|c|}{$\begin{array}{l}\text { Multivariate analysis } \\
\qquad(\mathrm{n}=407)^{\star}\end{array}$} & \multicolumn{2}{|c|}{$\begin{array}{c}\text { Multivariate analysis except } \\
\text { nodal-blocking drugs }(n=337)^{\dagger}\end{array}$} \\
\hline & $P$ value & HR (95\% Cl) & $P$ value & HR (95\% Cl) & $P$ value & HR $(95 \% \mathrm{Cl})$ \\
\hline Age $(<61$ years $=1)$ & 0.04 & $1.55(1.02-2.36)$ & 0.18 & $1.37(0.86-2.20)$ & 0.16 & $1.47(0.86-2.55)$ \\
\hline Sex $($ female $=1)$ & 0.19 & $1.38(0.86-2.33)$ & 0.67 & $1.12(0.67-1.95)$ & 0.30 & $1.37(0.76-2.57)$ \\
\hline Heart rate $(<66$ beats $/ \min =1)$ & 0.21 & $1.30(0.86-1.97)$ & 0.26 & $1.29(0.83-2.01)$ & 0.29 & $1.34(0.88-2.08)$ \\
\hline $\begin{array}{l}\text { Incremental rate of QRS duration } \\
(<3 \mathrm{~ms} / \text { year }=1)\end{array}$ & $<0.0001$ & $2.52(1.66-3.87)$ & 0.01 & $1.79(1.15-2.80)$ & 0.01 & $2.08(1.25-3.53)$ \\
\hline $\begin{array}{l}\text { Incremental rate of PR interval } \\
(<2 \mathrm{~ms} / \text { year }=1)\end{array}$ & $<0.0001$ & $3.39(2.22-5.23)$ & 0.001 & $2.25(1.42-3.58)$ & 0.002 & $2.34(1.36-4.05)$ \\
\hline
\end{tabular}

*Adjusted for age, sex, heart rate, arrhythmia except AF, nodal-blocking drugs, incremental rate of QRS duration, incremental rate of PR interval, diabetes mellitus, ischemic heart disease, and cardiomyopathy.

${ }^{\dagger}$ Adjusted for age and sex, heart rate, arrhythmia except AF, incremental rate of QRS duration, incremental rate of PR interval, diabetes mellitus, ischemic heart disease, and cardiomyopathy.

$\mathrm{HR}$, hazard ratio; $\mathrm{Cl}$, confidence interval. Other abbreviations as in Table 1.

tics of the QRS complex in the baseline and follow-up ECGs. Heart rate was significantly slower in the follow-up ECG than in the baseline ECG. PR interval and QRS duration significantly increased by $8.4 \%$ and $20.2 \%$, respectively, during follow-up. In the baseline ECG, normal QRS duration was present in $31.2 \%$ of the study population, and right bundle branch block (RBBB), RBBB with left anterior hemiblock (LAH), left bundle branch block (LBBB), or nonspecific intraventricular conduction disturbance (NSIVCD) was present in the remaining patients. During the follow-up, RBBB, RBBB with LAH, and LBBB developed in 99 (21.6\%), 9 (2.0\%), and 22 (4.8\%), respectively, of the patients who showed normal QRS duration in the baseline ECG. The prevalence of RBBB with or without LAH was significantly higher in the follow-up ECG than in the baseline ECG. The prevalence of LBBB showed a trend to increase during the follow-up, but the prevalence of NSIVCD did not significantly alter.

\section{Long-Term Prognosis}

HF The most common cause of the endpoints was HF hospitalization. During the follow-up period, 109 patients were hospitalized because of HF. At baseline, age was significantly higher in patients with HF hospitalization than in those without $(60.7 \pm 11.2$ vs. $57.0 \pm 15.5$ years; $\mathrm{P}=0.02)$, but the sex prevalence was not significantly different between the 2 groups ( $76 \%$ vs. $74 \%$ male; $\mathrm{P}=0.64$ ). The prevalence of ischemic heart disease, cardiomyopathy, valvular heart disease, and various arrhythmias excluding atrial fibrillation (AF) was significantly higher in patients with HF hospitalization than in those without $(\mathrm{P}<0.05$ for each). The prevalence of diabetes and $\mathrm{AF}$ was marginally higher in patients with HF hospitalization than in those without ( $\mathrm{P}=0.10$ and 0.08 , respectively). The follow-up period was not significantly different between patients with and without $\mathrm{HF}$ hospitalization $(13.8 \pm 6.2$ vs. $13.2 \pm 6.5$ years, $\mathrm{P}=0.37$ ). Table $\mathrm{S} 1$ shows the ECG characteristics. In the baseline ECG, heart rate was significantly faster and QRS duration significantly shorter in patients with HF hospitalization than in those without, but the PR interval did not differ between patients with and without HF hospitalization. The prevalence of LBBB tended to be higher in patients with HF hospitalization than in those without, whereas for RBBB it was the reverse. The temporal increase in PR interval was significantly longer and the incremental rate of the PR interval was significantly higher in patients with HF hospitalization than in those without. The temporal increase in QRS duration was significantly longer and the incremental rate of QRS duration was significantly higher in patients with HF hospitalization than in those without.

Of the total patients enrolled, 43 and 8, respectively, developed AF and second- or third-degree atrioventricular block during the follow-up, and the PR interval failed to be measured in them, so they were excluded when the long-term prognosis was evaluated. Therefore, the final study sample in this analysis consisted of 407 patients (men, 300 [73.7\%]; mean age, 57.4 \pm 15.1 years). Figure 1 shows the Kaplan-Meier estimates of the probability of freedom from HF hospitalization. The temporal incremental rate of QRS duration of $3 \mathrm{~ms} / \mathrm{year}$ or greater was associated with a significantly increased risk of $\mathrm{HF}$ hospitalization than that of $<3 \mathrm{~ms} /$ year (hazard ratio [HR], 2.5; 95\% confidence interval [CI], 1.66-3.87; $\mathrm{P}<0.0001$ ) (Figure 1A). The temporal incremental rate of PR interval of $2 \mathrm{~ms} /$ year or greater was associated with a significantly increased risk of $\mathrm{HF}$ hospitalization than that of $<2 \mathrm{~ms} /$ year $(\mathrm{HR}$, 3.4; 95\% CI, 2.22-5.23; $\mathrm{P}<0.0001$ ) (Figure 1B). Furthermore, when present together, the temporal incremental rate of QRS duration and PR interval potentiated each other, leading to much higher rate of hospitalization for $\mathrm{HF}$ (HR, 6.0; 95\% CI, 3.45-10.67; $\mathrm{P}<0.0001$ ) (Figure $1 \mathrm{C}$ ). Table 3 shows the univariate and multivariate analyses in association with HF hospitalization. The temporal incremental rate of QRS duration and PR interval was significantly associated with HF hospitalization, even after exclusion of nodal-blocking drugs. Both ECG variables were independent of age, sex, and heart rate. Confounding diseases were not associated with HF hospitalization.

Cardiovascular Mortality A total of 16 patients died of cardiovascular causes during the follow-up: 12 from HF, 2 from ventricular tachyarrhythmia, 1 from cerebral hemorrhage, and 1 from occlusion of the celiac artery. At baseline, age was not significantly different between patients with and without cardiovascular death $(63.3 \pm 11.8$ vs. $57.7 \pm 14.7$ years; $\mathrm{P}=0.14)$, nor was the sex prevalence between the 2 groups ( $88 \mathrm{vs} .74 \%$ male; $\mathrm{P}=0.19$ ). The prevalence of cardiomyopathy was significantly higher in patients with cardiovascular death than in those without ( 18.8 vs. $2.5 \% ; \mathrm{P}=0.01$ ), but the prevalence of ischemic heart disease, valvular heart disease, $\mathrm{AF}$, and diabetes was not significantly different between the 2 groups. The prevalence of various arrhythmias excluding AF was marginally higher in patients with cardiovascular death than in those without $(\mathrm{P}=0.09)$. The follow-up period was not significantly different between patients with and without cardiovascular death (10.9 \pm 4.9 vs. $13.4 \pm 6.4$ years, $\mathrm{P}=0.13)$. Table $\mathbf{S} 2$ shows 
A

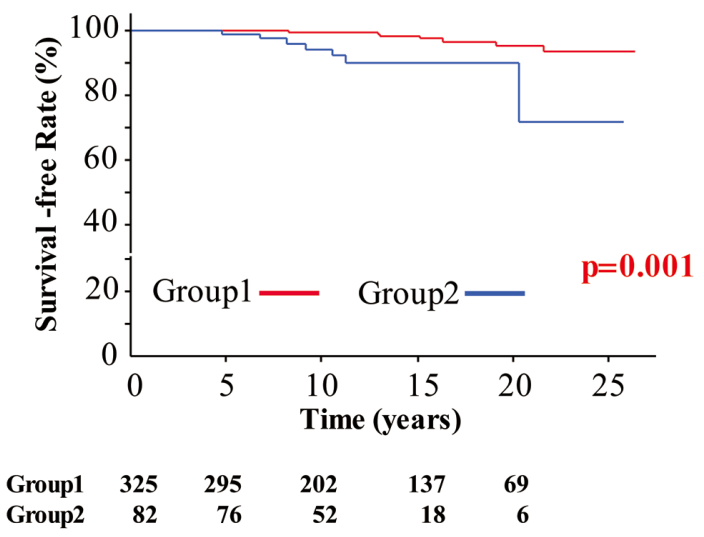

B

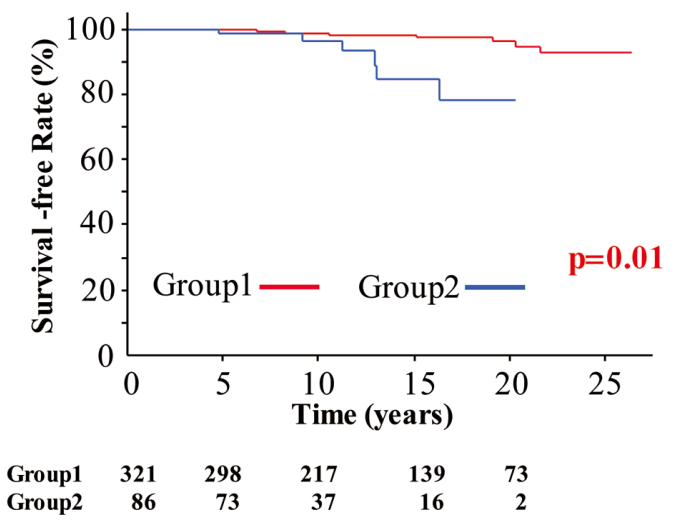

Figure 2. Kaplan-Meier survival analysis for cardiovascular mortality. (A) QRS-duration incremental rate $<5$ ms/year (Group 1 ) vs. $\geq 5 \mathrm{~ms} /$ year (Group 2). (B) PR-interval incremental rate $<4 \mathrm{~ms} /$ year (Group 1) vs. $\geq 4 \mathrm{~ms} /$ year (Group 2). Numbers of subjects at risk are shown under each graph.

\begin{tabular}{|c|c|c|c|c|c|c|}
\hline & \multicolumn{2}{|c|}{$\begin{array}{l}\text { Univariate analysis } \\
\qquad(n=407)\end{array}$} & \multicolumn{2}{|c|}{$\begin{array}{l}\text { Multivariate analysis } \\
\qquad(\mathrm{n}=407)^{\star}\end{array}$} & \multicolumn{2}{|c|}{$\begin{array}{c}\text { Multivariate analysis except } \\
\text { nodal-blocking drugs }(n=337)^{\dagger}\end{array}$} \\
\hline & $P$ value & HR (95\% Cl) & $P$ value & HR $(95 \% \mathrm{Cl})$ & $P$ value & HR (95\% Cl) \\
\hline Age $(<61$ years $=1)$ & 0.10 & $2.44(0.86-7.50)$ & 0.30 & $1.82(0.59-5.95)$ & 0.97 & $1.02(0.27-3.92)$ \\
\hline Sex $($ female $=1)$ & 0.17 & $2.56(0.71-16.37)$ & 0.16 & $2.72(0.71-18.00)$ & 0.49 & $1.81(0.37-14.13)$ \\
\hline Heart rate $(<66$ beats $/ \min =1)$ & 0.98 & $1.01(0.35-2.82)$ & 0.54 & $0.71(0.23-2.13)$ & 0.67 & $1.34(0.34-5.41)$ \\
\hline $\begin{array}{l}\text { Incremental rate of QRS duration } \\
(<5 \mathrm{~ms} / \text { year }=1)\end{array}$ & 0.004 & $5.04(1.72-14.49)$ & 0.06 & $3.18(0.95-10.61)$ & 0.03 & $4.31(1.19-16.50)$ \\
\hline $\begin{array}{l}\text { Incremental rate of PR interval } \\
(<4 \mathrm{~ms} / \text { year }=1)\end{array}$ & 0.005 & $5.63(1.76-17.62)$ & 0.17 & $2.39(0.69-8.21)$ & 0.02 & $6.90(1.47-36.96)$ \\
\hline
\end{tabular}

${ }^{*}$ Adjusted for age, sex, heart rate, LBBB, arrhythmias except AF, incremental rate of QRS duration, incremental rate of PR interval, diabetes mellitus, and cardiomyopathy.

†Adjusted for age, sex, heart rate, arrhythmias except AF, incremental rate of QRS duration, incremental rate of PR interval, and cardiomyopathy. Abbreviations as in Tables 1-3.

the ECG characteristics. In the first ECG, heart rate, PR interval, and QRS duration did not differ between patients with and without cardiovascular death. The prevalence of LBBB was significantly higher in patients with cardiovascular death than in those without; but the prevalence of other abnormal intraventricular conductions did not differ between the 2 groups. The temporal increase in PR interval and the incremental rate of PR interval were significantly greater in patients with cardiovascular death than in those without. The temporal increase in QRS duration was not significantly different between patients with and without cardiovascular death, but the incremental rate of QRS duration was significantly higher in patients with cardiovascular death than in those without.

Long-term prognosis was analyzed after excluding patients who developed AF and second- or third-degree atrioventricular block, being evaluated in 407 patients. In Figure 2, the Kaplan-Meier estimates of the probability of freedom from cardiovascular mortality indicate that the temporal incremental rate of QRS duration of $5 \mathrm{~ms} /$ year or greater was associated with a significantly increased risk of cardiovascular mortality than that of $<5$ ms/year (HR, 5.0; 95\% CI, 1.72-14.49; P=0.004) (Figure 2A). The temporal incremental rate of PR interval of
$4 \mathrm{~ms} /$ year or greater was associated with a significantly increased risk of cardiovascular mortality than that of $<4$ ms/year (HR, 5.6; 95\% CI, 1.76-17.62; $\mathrm{P}=0.005$ ) (Figure 2B). Table 4 shows the univariate and multivariate survival analyses in association with cardiovascular death. The temporal incremental rate of QRS duration and PR interval was significantly associated with cardiovascular mortality after exclusion of nodal-blocking drugs. Both ECG variables were independent of age, sex, and heart rate. Confounding diseases were not associated with cardiovascular mortality.

All-Cause Mortality A total of 59 patients (12.9\%) died during the follow-up: 16 from cardiovascular death as noted earlier, 24 from malignant disorders, 6 from severe infection, and 13 from other causes. At baseline, age was significantly higher in patients with all-cause death than in those without ( $63.4 \pm 10.8$ vs. $57.1 \pm 15.0$ years; $\mathrm{P}=0.002$ ), but the sex prevalence was not significantly different between the 2 groups ( $80 \%$ vs. $74 \%$ male; $\mathrm{P}=0.32$ ). The prevalences of cardiomyopathy and malignant disease were significantly higher in patients with all-cause death than in those without $(\mathrm{P}<0.05$ for each), but the prevalences of ischemic heart disease, valvular heart disease, diabetes, and AF were not significantly different 
Table 5. Univariate and Multivariate Analyses of All-Cause Death

\begin{tabular}{|c|c|c|c|c|c|c|}
\hline & \multicolumn{2}{|c|}{$\begin{array}{l}\text { Univariate analysis } \\
\qquad(n=407)\end{array}$} & \multicolumn{2}{|c|}{$\begin{array}{l}\text { Multivariate analysis } \\
\qquad(\mathrm{n}=407)^{\star}\end{array}$} & \multicolumn{2}{|c|}{$\begin{array}{c}\text { Multivariate analysis except } \\
\text { nodal-blocking drugs }(n=337)^{\dagger}\end{array}$} \\
\hline & $P$ value & $\mathrm{HR}(95 \% \mathrm{Cl})$ & $P$ value & $\mathrm{HR}(95 \% \mathrm{Cl})$ & $P$ value & $\mathrm{HR}(95 \% \mathrm{Cl})$ \\
\hline Age $(<61$ years $=1)$ & $<0.0001$ & $3.40(1.89-6.35)$ & 0.0002 & $3.36(1.76-6.65)$ & 0.01 & $2.64(1.30-5.47)$ \\
\hline Sex $($ female $=1)$ & 0.44 & $1.28(0.69-2.56)$ & 0.32 & $1.40(0.73-2.84)$ & 0.17 & $1.68(0.81-3.84)$ \\
\hline Heart rate $(<66$ beats $/ \min =1)$ & 0.50 & $1.21(0.70-2.11)$ & 0.39 & $1.29(0.72-2.32)$ & 0.52 & $1.24(0.65-2.37)$ \\
\hline $\begin{array}{l}\text { Incremental rate of QRS duration } \\
(<5 \mathrm{~ms} / \text { year }=1)\end{array}$ & 0.004 & $2.59(1.38-4.66)$ & 0.05 & $1.95(0.99-3.66)$ & - & - \\
\hline $\begin{array}{l}\text { Incremental rate of PR interval } \\
(<4 \mathrm{~ms} / \text { year }=1)\end{array}$ & 0.003 & $2.92(1.48-5.49)$ & 0.39 & $1.37(0.65-2.75)$ & 0.15 & $1.87(0.79-4.19)$ \\
\hline Malignant disease (no malignancy $=1$ ) & 0.004 & $4.09(1.65-8.78)$ & 0.02 & $3.27(1.25-7.47)$ & 0.02 & $4.03(1.41-9.97)$ \\
\hline
\end{tabular}

*Adjusted for age, sex, heart rate, AF, nodal-blocking drugs, incremental rate of QRS duration, incremental rate of PQ interval, cardiomyopathy, and malignant disease.

${ }^{\dagger}$ Adjusted for age, sex, heart rate, incremental rate of $P Q$ interval, cardiomyopathy, and malignant disease.

Abbreviations as in Tables 1,3.

between the 2 groups. The follow-up period was marginally different between patients with and without all-cause death $(11.8 \pm 5.9$ vs. $13.5 \pm 6.4$ years, $\mathrm{P}=0.05)$. Table $\mathrm{S} 3$ shows the $\mathrm{ECG}$ characteristics. In the baseline ECG, heart rate, PR interval, and QRS duration was not significantly different between patients who died and those who survived. The prevalence of abnormal intraventricular conduction was not significantly different between patients who died and survived. The temporal increase in PR interval and the incremental rate of PR interval was not significantly different between patients who died and those who survived. The temporal increase in QRS duration was not significantly different between patients who died and those who survived, but the incremental rate of QRS duration was significantly higher in patients who died than in those who survived.

Long-term prognosis was analyzed after excluding the patients who developed AF and second- or third-degree atrioventricular block, being evaluated in 407 patients. Table 5 shows the univariate and multivariate survival analyses in association with all-cause death. Advanced age and malignant diseases were independently associated with all-cause mortality even after exclusion of nodal-blocking drugs. Neither the temporal incremental rate of QRS duration nor the temporal incremental rate of PR interval was independently associated with cardiovascular mortality. In addition, sex, heart rate, and confounding diseases except malignancy were not associated with all-cause mortality.

\section{Discussion}

Historically, in the 19th century Adams and Stokes separately reported a patient who suffered fainting episodes with slow pulse rate. Congenital heart block and familial occurrence of atrioventricular conduction block were subsequently noted, ${ }^{\mathbf{8}, 9}$ suggesting inherited conduction disorders. PCCD was initially reported as an electrical conduction disorder. ${ }^{10}$ Familial clustering of conduction system degeneration has led to the discovery of novel mutations resulting in PCCD in the absence of structural heart disease. ${ }^{11}$ To date, multiple molecular defects responsible for PCCD have been found. ${ }^{12}$ In addition, congenital heart diseases are accompanied by functional abnormalities of the conduction system,,${ }^{13}$ and overlap of PCCD with structural heart diseases such as cardiomyopathy has been reported. ${ }^{14,15}$ Neuromuscular disorders are also associated with the development of PCCD. ${ }^{16}$ Taken together, PCCD appears not to be a single clinical entity caused by a single- gene mutation. We expand the concept of PCCD to include various cardiac diseases. In our large hospital-based population, the major findings of PCCD are summarizes as follows: (1) the temporal incremental rate of PR interval was independently associated with increased risk of HF hospitalization and cardiovascular mortality, (2) the temporal incremental rate of QRS duration was independently associated with increased risk of HF hospitalization and cardiovascular mortality, and thus (3) the severity of PCCD was intimately associated with an adverse prognosis.

\section{Pathophysiology of PCCD}

The cardiac impulse originating from the sinus node activates the atria and the ventricle, with the specialized conduction system depolarized first and the working myocardium secondarily depolarized. For impulse propagation in both the atrium and ventricle, the voltage-gated sodium channel and gap junctions are of major importance, because the former determines the excitability of the cells and the latter serves as the depolarizing current transmitted from cell to cell. Lev and Unger ${ }^{1}$ and Lenègre $^{2}$ reported the association of excessive interstitial fibrosis near and in the specialized conduction system with PR-interval prolongation and QRS-duration widening in postmortem examinations. Their findings suggested that interstitial fibrosis played a significant role in activation delay in patients with PCCD. Familial clustering of cardiac conduction disturbance, which occur in the absence of structural heart disease or systemic disease, results from mutations in cardiac ion channel genes and associated or modifying proteins such as cytoskeletal proteins. The SCN5A mutation that results in nonfunctional human cardiac sodium channels has been found in familial PCCD. ${ }^{17}$ This mutation consequently gives rise to slowing cardiac conduction. Besides, SCN5A has been reported as associated with dilated cardiomyopathy. ${ }^{15}$ Therefore, structural and functional PCCDs may share the same pathophysiological background. The temporal incremental rate of QRS duration may correlate with deterioration of ventricular function, leading to increased LV filling pressures and consequently myocardial ischemia. Such longstanding conditions also may result in subendocardial fibrosis. ${ }^{18}$ Thus, progressive widening of the QRS duration could be a surrogate for perpetual deterioration of the LV. Consistent with these pathophysiological mechanisms, the major outcome of this study was HF hospitalization.

Although most reports dealing with PCCD have consisted of small groups of patients, the presence of mutations in the 
SCN5A, PRKAG2, NKX2-5 and LMNA genes, which were found to be linked to cardiomyopathies, conduction disease, and ventricular arrhythmias, has been investigated. Inter- and intraventricular conduction disturbances are the most common ECG pattern preceding the development of complete heart block, particularly in the presence of structural heart disease. Recently, it was reported that gain-of-function mutations in TRPM4 encoding a calcium-activated nonselective cation channel of the transient receptor potential melastatin (TRPM) ion channel cause autosomal dominant isolated cardiac conduction disease. ${ }^{19}$ In the present study, the temporal deterioration in supraventricular and ventricular conduction was independently associated with a worse prognosis of PCCD. Although gene analysis was not performed in this study, further investigation is needed to clarify whether gene mutation exists to stratify the risk of PCCD in our cohort.

\section{Prognosis}

General Population In the Framingham study, ${ }^{20}$ the prevalence of BBB was related to aging and was more common among men than among women. Some studies report that there is no significant relation between $\mathrm{BBB}$ and mortality in the general population. ${ }^{21-24}$ Regarding newly occurred BBB, left BBB was associated with an increased risk of developing overt cardiovascular disease and increased cardiac mortality. ${ }^{25,26}$ These studies suggest that progressive degeneration of the cardiac myocardium might coincide with the development of BBB.

Medical Population In the medical population, prolonged QRS duration is reported to be a significant and independent predictor of cardiovascular mortality. ${ }^{4}$ Several studies have shown that both left and right BBB are associated with increased mortality among patients with structural heart diseases. ${ }^{27-29}$ In myocardial infarction, patients with BBB presented significantly more often with congestive HF and higher mortality than do their counterparts without BBB. ${ }^{30}$ Prolonged QRS duration is also associated with decreased left ventricular systolic function and poor prognosis in patients with coronary artery disease. ${ }^{6}$ These studies indicate that QRS duration matters a great deal for the prognosis of patients with cardiovascular diseases.

Though the follow-up period was not long, serial ECG recordings have revealed that an increase in QRS duration is associated with adverse outcomes in patients with HF. ${ }^{31,32}$ Biventricular pacing therapy for HF that corrected the mechanical dyssynchrony caused by ventricular conduction disturbance provides evidence that patients with prolonged QRS duration have a higher susceptibility to improving their physical status by cardiac resynchronization therapy. ${ }^{33}$ Along with these studies, the present study elucidated that the temporal incremental rate of QRS duration was independently associated with long-term poor prognosis. In addition, this study underscored the independent association between the temporal incremental rate of PR interval and QRS duration. Patients who developed AF during the follow-up were not enrolled in this study, because serial change in the PR interval was not able to be measured in those patients. Therefore, the results of this study might underestimate the prognosis of PCCD, because AF can be regarded as advanced stage of atrial conduction delay.

In this hospital-based cohort, the endpoints were determined by analysis of detailed medical records in which the prognostic outcomes were identified according to ICD codes, so our data were reliable during the long-term follow-up period. In addition, medical records are useful for evaluating physical conditions not only at 1 time point but also during follow-up, thus we precisely reviewed the medical records of all patients enrolled throughout the follow-up period.

\section{Study Limitations}

Because this cohort study was not prospectively conducted using ECG recordings, several limitations are inherent. First, we determined the increase of the PR interval and QRS duration by averaging their temporal variations. Those increases might not be temporally constant. To avoid excess change during a short time, we used ECGs for which the recording interval was more than 1 year. Second, the temporal increment rate of the PR interval was a few milliseconds. Although the temporal increment rate of the PR interval was used to stratify the prognostic outcomes, the effect of autonomic tone on the PR interval was not negligible. Third, intermittent BBB might have been missed unless the ECG finding was recorded in the hospital. Because no intermittent BBB could be documented during the follow-up in patients enrolled in this study, it is thought that intermittent BBB rarely occurred in this hospitalbased population. Fourth, we did not involve cardiac functions such as left ventricular ejection fraction and mitral regurgitation. Lastly, because our study included patients who underwent ECG recording in our hospital, the risk of HF hospitalization in the study population undoubtedly was greater than that in the general population. Therefore, this factor should be considered when our results are extrapolated to a broader population.

\section{Study Implications}

PCCD was primarily identified as a cardiac electrical disease representing temporal prolongation of the QRS duration. Although prolonged QRS duration is known to be associated with adverse prognosis, the prognosis of PCCD remains unclear in a large population. This study demonstrated that patients with a higher degree of PCCD have a worse prognosis. The independent relationship between PCCD and HF hospitalization and between PCCD and cardiovascular mortality helps find patients who are at risk. The increased risk of HF hospitalization associated with the temporal incremental rate of QRS duration suggests a possible role for cardiac resynchronization therapy, particularly among patients with systolic HF. Therefore, our results indicate that careful follow-up of the ECG deserves attention for determining which patients are most likely to benefit from preventive HF therapy such as diuretics. For example, when patients visit cardiology clinics, repetitive ECG recordings might provide a noninvasive means of identifying those at high risk for worsening of HF. To make the temporal variation of the PR interval and QRS duration clinically available for treatment of patients at the early stage, improvements in the software for automatic display of these ECG variables are needed.

\section{Acknowledgments}

The authors thank Seiichi Fujisaki, Akihiko Ejima, and Tatsumi Uchiyama (GE Yokokawa Medical System Co) for their technical assistance.

\section{Disclosures}

None.

\section{References}

1. Lev M, Unger PN. The pathology of the conduction system in acquired heart disease. I: Severe atrioventricular block. AMA Arch Pathol 1955; 60: 502-529.

2. Lenegre J. Etiology and pathology of bilateral bundle branch block 
in relation to complete heart block. Prog Cardiovasc Dis 1964; 6: 409-444.

3. Holm H, Gudbjartsson DF, Arnar DO, Thorleifsson G, Thorgeirsson $\mathrm{G}$, Stefansdottir $\mathrm{H}$, et al. Several common variants modulate heart rate, PR interval and QRS duration. Nat Genet 2010; 42: 117-122.

4. Desai AD, Yaw TS, Yamazaki T, Kaykha A, Chun S, Froelicher VF. Prognostic significance of quantitative QRS duration. Am J Med 2006; 119: $600-606$

5. Bode-Schnurbus L, Bocker D, Block M, Gradaus R, Heinecke A, Breithardt G, et al. QRS duration: A simple marker for predicting cardiac mortality in ICD patients with heart failure. Heart 2003; 89: $1157-1162$.

6. Murkofsky RL, Dangas G, Diamond JA, Mehta D, Schaffer A, Ambrose JA. A prolonged QRS duration on surface electrocardiogram is a specific indicator of left ventricular dysfunction [see Comment]. J Am Coll Cardiol 1998; 32: 476-482.

7. Moss AJ, Zareba W, Hall WJ, Klein H, Wilber DJ, Cannom DS, et al. Prophylactic implantation of a defibrillator in patients with myocardial infarction and reduced ejection fraction. N Engl J Med 2002; 346: $877-883$.

8. Trevino AJ, Beller BM. Conduction disturbances of the left bundle branch system and their relationship to complete heart block. II: A review of differential diagnosis, pathology and clinical significance. Am J Med 1971; 51: 374-382.

9. Schaal SF, Seidensticker J, Goodman R, Wooley CF. Familial right bundle-branch block, left axis deviation, complete heart block, and early death: A heritable disorder of cardiac conduction. Ann Intern Med 1973; 79: 63-66.

10. Smits JP, Veldkamp MW, Wilde AA. Mechanisms of inherited cardiac conduction disease. Europace 2005; 7: 122-137.

11. Benson DW, Wang DW, Dyment M, Knilans TK, Fish FA, Strieper $\mathrm{MJ}$, et al. Congenital sick sinus syndrome caused by recessive mutations in the cardiac sodium channel gene (SCN5A). J Clin Invest 2003; 112: 1019-1028.

12. Wolf CM, Berul CI. Inherited conduction system abnormalities: One group of diseases, many genes. J Cardiovasc Electrophysiol 2006; 17: $446-455$.

13. Schott JJ, Benson DW, Basson CT, Pease W, Silberbach GM, Moak $\mathrm{JP}$, et al. Congenital heart disease caused by mutations in the transcription factor NKX2-5. Science 1998; 281: 108-111.

14. Nguyen TP, Wang DW, Rhodes TH, George AL Jr. Divergent biophysical defects caused by mutant sodium channels in dilated cardiomyopathy with arrhythmia. Circ Res 2008; 102: 364-371.

15. Wilde AA, Brugada R. Phenotypical manifestations of mutations in the genes encoding subunits of the cardiac sodium channel. Circ Res 2011; 108: 884-897.

16. Bonne G, Di Barletta MR, Varnous S, Becane HM, Hammouda EH, Merlini L, et al. Mutations in the gene encoding lamin A/C cause autosomal dominant Emery-Dreifuss muscular dystrophy. Nat Genet 1999; 21: 285-288.

17. Schott JJ, Alshinawi C, Kyndt F, Probst V, Hoorntje TM, Hulsbeek M, et al. Cardiac conduction defects associate with mutations in SCN5A. Nat Genet 1999; 23: 20-21.

18. Aoki T, Fukumoto Y, Sugimura K, Oikawa M, Satoh K, Nakano M, et al. Prognostic impact of myocardial interstitial fibrosis in nonischemic heart failure: Comparison between preserved and reduced ejection fraction heart failure. Circ J 2011; 75: 2605-2613.

19. Liu H, El Zein L, Kruse M, Guinamard R, Beckmann A, Bozio A, et al. Gain-of-function mutations in TRPM4 cause autosomal dominant isolated cardiac conduction disease. Circ Cardiovasc Genet 2010; 3: $374-385$

20. Kreger BE, Anderson KM, Kannel WB. Prevalence of intraventricular block in the general population: The Framingham study. Am Heart J 1989; 117: 903-910.
21. Eriksson P, Hansson PO, Eriksson H, Dellborg M. Bundle-branch block in a general male population: The study of men born 1913. Circulation 1998; 98: 2494-2500.

22. Fleg JL, Das DN, Lakatta EG. Right bundle branch block: Long-term prognosis in apparently healthy men. J Am Coll Cardiol 1983; 1: $887-892$.

23. Liao YL, Emidy LA, Dyer A, Hewitt JS, Shekelle RB, Paul O, et al. Characteristics and prognosis of incomplete right bundle branch block: An epidemiologic study. J Am Coll Cardiol 1986; 7: 492-499.

24. Fahy GJ, Pinski SL, Miller DP, McCabe N, Pye C, Walsh MJ, et al. Natural history of isolated bundle branch block. Am J Cardiol 1996; 77: $1185-1190$.

25. Schneider JF, Thomas HE Jr, Kreger BE, McNamara PM, Kannel WB. Newly acquired left bundle-branch block: The Framingham study. Ann Intern Med 1979; 90: 303-310.

26. Schneider JF, Thomas HE, Kreger BE, McNamara PM, Sorlie P, Kannel WB. Newly acquired right bundle-branch block: The Framingham study. Ann Intern Med 1980; 92: 37-44.

27. Hindman MC, Wagner GS, JaRo M, Atkins JM, Scheinman MM, DeSanctis RW, et al. The clinical significance of bundle branch block complicating acute myocardial infarction. 1: Clinical characteristics, hospital mortality, and one-year follow-up. Circulation 1978; 58: 679-688.

28. Ricou F, Nicod P, Gilpin E, Henning H, Ross J Jr. Influence of right bundle branch block on short- and long-term survival after acute anterior myocardial infarction. J Am Coll Cardiol 1991; 17: 858-863.

29. Hesse B, Diaz LA, Snader CE, Blackstone EH, Lauer MS. Complete bundle branch block as an independent predictor of all-cause mortality: Report of 7,073 patients referred for nuclear exercise testing. Am JMed 2001; 110: 253-259.

30. Melgarejo-Moreno A, Galcera-Tomas J, Garcia-Alberola A, ValdesChavarri M, Castillo-Soria FJ, Mira-Sanchez E, et al. Incidence, clinical characteristics, and prognostic significance of right bundle-branch block in acute myocardial infarction: A study in the thrombolytic era. Circulation 1997; 96: 1139-1144.

31. Shamim W, Yousufuddin M, Cicoria M, Gibson DG, Coats AJ, Henein MY. Incremental changes in QRS duration in serial ECGs over time identify high risk elderly patients with heart failure. Heart 2002; 88: $47-51$.

32. Grigioni F, Carinci V, Boriani G, Bracchetti G, Potena L, Magnani G, et al. Accelerated QRS widening as an independent predictor of cardiac death or of the need for heart transplantation in patients with congestive heart failure. J Heart Lung Transplant 2002; 21: 899902.

33. Moss AJ. What we have learned from the family of multicenter automatic defibrillator implantation trials. Circ J 2010; 74: 1038-1041.

\section{Supplementary Files}

Supplementary File 1

Figure S1. Temporal alterations of 12-lead ECGs in a patient with progressive cardiac conduction disease (PCCD).

Table S1. Comparison of Baseline ECG and Follow-up ECG According to Heart Failure Hospitalization

Table S2. Comparison of Baseline ECG and Follow-up ECG According to Cardiovascular Death

Table S3. Comparison of Baseline ECG and Follow-up ECG According to All-Cause Death

Please find supplementary file(s);

http://dx.doi.org/10.1253/circj.CJ-12-0849 